\title{
Assessment of visual function in suspected ocular malingering
}

\author{
M FAHLE AND G MOHN \\ From the Department of Pathophysiology of Vision and Neuro-ophthalmology, University Eye Clinic, \\ Tübingen, West Germany
}

SUMMARY Three new techniques are presented for assessing visual acuity in the presence of suspected malingering. The first technique is based on the preferential looking method commonly used to test acuity in infants. The second uses polarising filters to present stimuli briefly to both eyes or to each eye alone, without the patient's being aware of which eye is tested. The third presents the stimuli on a computer monitor and separates the ray paths of the two eyes by means of special spectacles that obscure vision in one eye for fractions of a second while a stimulus is presented.

Every now and then ophthalmologists are confronted with patients complaining of a functional visual loss for which no ready explanation can be found in their ophthalmological or neurological condition. When even the most thorough examination and questioning does not reveal a plausible cause of the loss or its reported severity, the ophthalmologist may eventually suspect ocular malingering. Of course the complaint must be considered to be true until proof to the contrary is obtained. Proof of ocular malingering, however, if possible at all, is often extremely difficult and time consuming.

Over the years a number of 'tricks' and special tests have been accumulated to help in the clarification of suspected cases of malingering. ${ }^{1-6}$ Here, we present three new techniques that have proved helpful in our clinic. The first technique is loosely based on the 'preferential looking' method designed several years ago to assess visual acuity in infants.' The second technique uses polarising filters to separate the stimuli to each eye, and flashed presentations to circumvent artefacts caused by eye blinking. The third technique is a modification of the second, using a personal computer and a pair of special spectacles which allow occlusion of each eye separately for fractions of a second.

\section{Material and methods}

PREFERENTIAL LOOKING

The first technique uses acuity cards normally used

Correspondence to Dr M Fahle, Center for Biological Information Processing, Massachusetts Institute of Technology. E25-201, Cambridge, Mass 02139 , USA. for assessing visual acuity in infants. ${ }^{89}$ The cards are rectangular pieces of grey cardboard $(C .30 \times 60 \mathrm{~cm})$ containing two circular targets of $9.5 \mathrm{~cm}$ diameter to the right and left of a small central peephole (Fig. 1). One of the targets is a grating pattern of vertical black-and-white stripes, with different stripe widths on different cards; the other appears uniformly grey but is actually made up of an extremely fine grating beyond the resolution limit of normal adults. The two targets are closely matched in colour and luminance.

For use with adult patients an additional circular coloured target of $7.5 \mathrm{~cm}$ diameter is placed in the centre of the card. The cards are presented at eye level through a horizontal opening $(20 \times 46 \mathrm{~cm})$ in a grey metal screen $(70 \times 170 \mathrm{~cm})$, with the grating target sometimes on the left and sometimes on the right side of the card. The examiner stands behind the screen while presenting the cards and can observe the patient through the peephole in the centre of the card. The patient sits at a distance of $1 \mathrm{~m}$ in front of the screen. At this distance the colour target subtends $4.2^{\circ}$ and the black-and-white targets $5.4^{\circ}$ with a centre-to-centre separation of $14^{\circ}$. The stripe width of the gratings varies from 66 to 0.5 minutes of arc in half-octave steps (equivalent to $20 / 1300$ to $20 / 10$ visual acuity; one octave is a doubling-or halvingof stripe width). Illumination is supplied by overhead incandescent lighting, producing a luminance around $30 \mathrm{~cd} / \mathrm{m}^{2}$ on the screen.

The patients are told they will undergo a colour vision test and that the perceived brightness of the colour is influenced by the patterns next to it. They are then asked to report the colour of the fixation target, which differs between cards, as well as the 


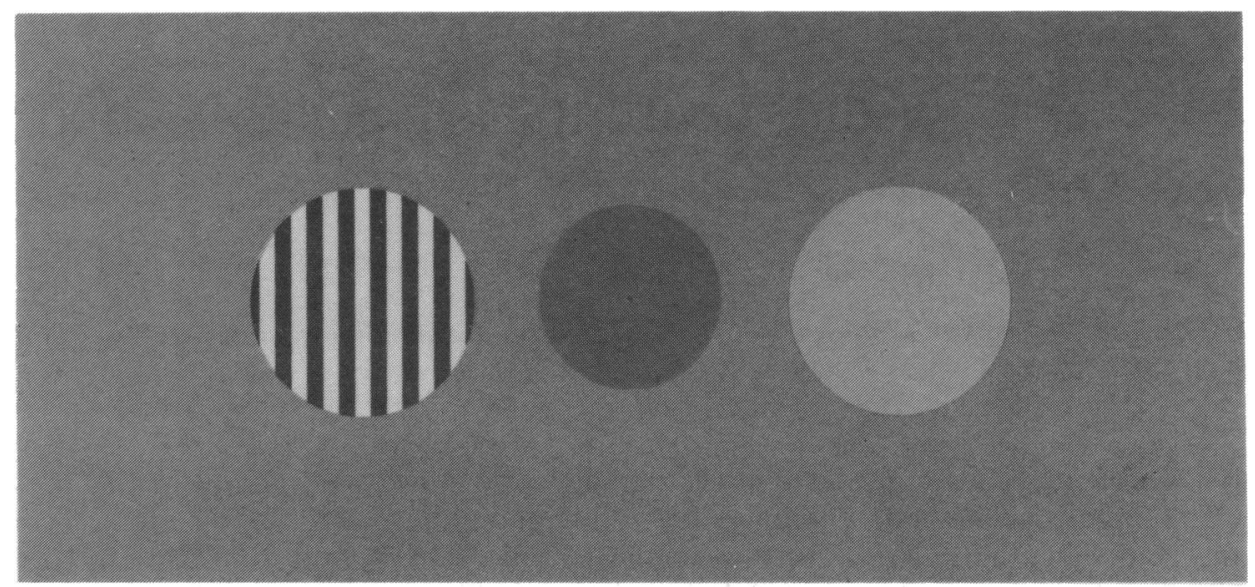

Fig. 1 Example of an acuity card with three circular targets. In this case the right target is uniformly grey, the left one shows a vertical grating pattern, and the middle one consists of a coloured spot. The grating and blank target are of equal mean luminance. A central small peep-hole is shown in the middle target.

position of the grating stimulus. The examiner, who takes care not to note the position of the grating target before presenting a card, then either verifies the patient's verbal report or makes a judgment of grating position on the basis of the patient's fixation pattern, since patients often look longer at the grating than at the uniformly grey target even when they report not seeing the stripes. By presenting gratings of different stripe widths the visual acuity is determined as the finest stripe width reported to be seen or, as in infants, as the finest grating for which the patient shows a fixation preference-assuming that as long as the grating target is preferentially fixated it is also resolved. Until now, no patient has realised that assessment of visual acuity rather than colour vision was the real aim of the examination.

\section{FLASH ACUITY}

For the second technique we used a standard Optotype projector equipped with three pieces of Polaroid mounted in front of the objective in a rotatable wheel. The investigator can switch between the filters, which are orientated so that two of them polarise light at right angles to each other, while the third is orientated between the other two. The light source of the projector consists either of the standard $20 \mathrm{~W}$ halogen bulb, providing a stimulus luminance of about $30 \mathrm{~cd} / \mathrm{m}^{2}$, or a commercially available flash gun $(60 \mathrm{~W})$. To facilitate the change between the two light sources they were mounted on a sliding adaptor which could be moved easily to bring one of the two sources into place.

The test stimuli are E-hooks - that is, the capital letter $E$ presented in one of four different orientations with the three short lines to the right or left or above or below the connecting line. These were chosen because, as with Landolt Cs, a 'new' stimulus of a given test size can be produced simply by rotating the one symbol, whereas several templates of each size would be required if different letters or numbers were used. The stimuli are presented singly, as the patients have to know exactly where the stimulus will appear during the very brief presentations, which are far too short for fixation eye movements (below $1 \mathrm{~ms}$ ).

The stimuli are projected on to an aluminised screen to ensure preservation of the plane of polarisation. The patient's head is stabilised by means of a head rest equipped with polarising filters matched to the two perpendicularly orientated filters in front of the projector. Thus the test stimuli can be flashed to both eyes or to either eye alone without the patient's being aware of which is the case. Further, tilting of the head does not diminish the separation between the ray paths of both eyes, as would have been the case with spectacles worn by the patient.

Incomplete separation of the stimuli of the two eyes by the polarising filters is another problem, common to all polarising tests. To overcome this problem we used polarising filters with very high extinction $(99.97 \%)$ in combination with a relatively high ambient luminance $\left(20 \mathrm{~cd} / \mathrm{m}^{2}\right)$. At the contrasts used, which were not far above threshold in the tested eye, the further reduction in mean luminance for the blocked eye led to strongly decreased resolution thresholds in this eye. Nevertheless, it is difficult to achieve an acuity decrease of more than one log unit in the blocked compared with the non-blocked condition. However, spurious identification of the stimulus through the blocked eye proved not to be a 


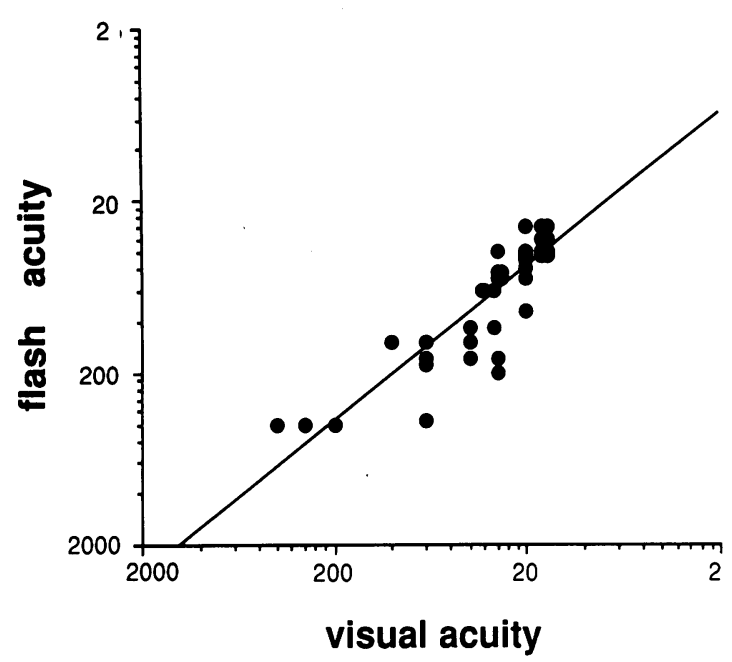

Fig. 2 Relation of flash acuity and standard two-point acuity in our set-up. Acuity is expressed as a dimensionless number (as in 6/6 or 20/20) where the number gives the numerator of the fraction while the denominator is always 20-for example, 20 corresponds to 20/20. Results are from 15 normal observers and 10 patients with different affections of the eye but without a history of malingering: cataract (four cases), glaucoma (three), keratoplastic (two), amblyopia (one). The regression line through the data is given by $0.4 x^{0.9}$; $r^{2}=0.77$.

serious problem, as we also attempted to reduce acuity in the better eye by addition of spherical lenses (positive dioptres).

The rationale is twofold: firstly, to prevent a stimulus presented to the affected eye from being recognised by the blocked, better eye owing to incomplete extinction. Secondly, this procedure makes the ease of recognition in both eyes similar by blurring the better eye in subjects with distinct interocular acuity differences. Thus the patients are deprived - at least partly - of an easy cue to discrimination between presentations to the better or the affected eye.

\section{FLASH ACUITY WITH A MONITOR}

The third technique is a more up-to-date version of the second. Here, a monitor under computer control (Atari ST) is used to present the stimuli, which could be of very different sizes, shapes, and orientations. Usually, E hooks are used, which are presented for a typical duration of $57 \mathrm{~ms}$ ( 4 frames). The patient wears a commercially available pair of spectacles designed for 3-D computer graphics, which are darkened for about $85 \mathrm{~ms}$ for one eye by trigger impulses from the computer while the test stimulus is presented. Alternatively, the eyes can be obscured alternately at a frequency of $35 \mathrm{~Hz}$ at counterphase so

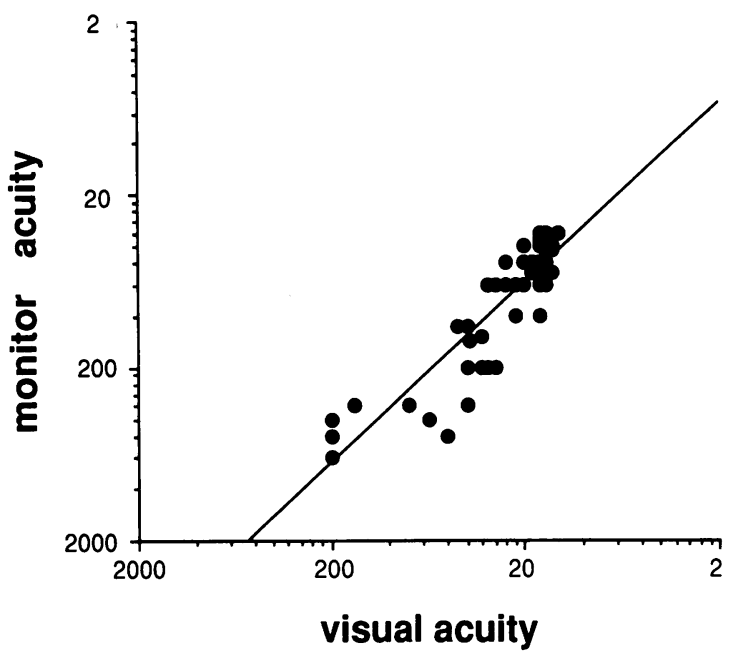

Fig. 3 Relation of monitor acuity and standard acuity in 16 normal observers and 11 patients. The patients suffered from cataract (four cases), glaucoma (two), diabetic retinopathy (one), keratoconus (one), perforating scleral injury (one), injury or herpes simplex of the cornea (two). The regression line is given by $0 \cdot 3 x^{10} ; r^{2}=0 \cdot 81$.

that each eye sees 35 frames per second. Then a stimulus can be shown to one eye for just one frame. Hence only one eye sees the stimulus, without the patient knowing which eye it was. With this method blocking was clearly better than with the polarising filters, resulting in an acuity decrease of the blocked eye by more than a factor of 70 . This means that even the largest stimulus cannot be identified through the blocked eye even if it has normal acuity.

The computer receives feedback about the patient's response by means of a joystick that is moved into a position according to the orientation of the $\mathrm{E}$ hook-either by the patient or by the investigator. According to whether the response is correct or not, the computer program then chooses the next stimulus size following a staircase procedure. Orientation is varied randomly and thresholds are calculated by probit analysis.

\section{Results and Discussion}

The first two of the above techniques have been used over the last year on a number of patients suspected of having better acuities than they were willing to admit. In some of them the results of our three tests proved the initial suspicion to be correct. In applying the tests, however, we also became aware of several shortcomings and limitations of the techniques. Firstly, the two flash acuity tests are not useful if both eyes are reported to have low visual acuity. Moreover, acuity is clearly lower with flash presentation 


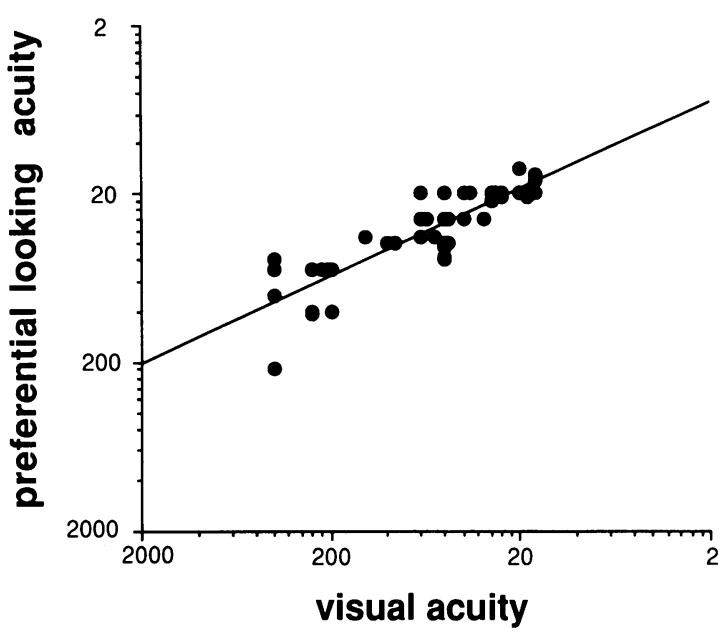

Fig. 4 Relation of preferential-looking acuity and standard acuity. Results of three normal observers and 23 patients. In some of the patients only one eye could be tested. The affections of the patients include cataract (11 cases), glaucoma (one), neuritis of the optic tract (one), pterygium (two), and amblyopia (one) as well as a tumour of the lid (one). The regression line is given by $1 \cdot 1 x^{0.25} ; r^{2}=0 \cdot 77$.

than with the much longer presentation times used in standard visual acuity testing. We therefore determined norm values for subjects with normal and degraded visual acuity (Figs. 2, 3). The results show that acuities measured by the flash acuity tests and standard acuity testing are closely correlated, making it possible to calculate standard visual acuity from the results of the flash acuity tests. The correlation coefficient (r) for the comparison between flash acuity and standard acuity is $\mathbf{0 . 8 8}$. The correlation between monitor acuity and standard acuity is even closer $(r=0 \cdot 9)$. Both correlations are significant beyond the $0 \cdot 1 \%$ range.
As to the preferential-looking method, this tends to fail if complete blindness is reported, as in the flash acuity test. But in general this technique has also proved to be valuable, and standard acuity can be inferred from the results obtained in normal subjects and in patients without a suspicion of malingering (Fig. 4). Again, the correlation between standard acuity and preferential looking acuity is highly significant, with a correlation coefficient of $r=0.88$.

In summary, despite some limitations, the three new techniques described here for the evaluation of visual acuity in uncooperative patients have proved to be successful tools in cases of suspected malingering.

This work was supported by DFG grants Fa $119 / 3-1$ and Fa 119/5-1 to MF and Fa 119/4-2 to GM. We thank H Weller for technical and secretarial help and M Repnow for writing the computer program.

\section{References}

1 Agatston H. Ocular malingering. Arch Ophthalmol 1944; 31 : 223 31 .

2 Baumgartner J, Epstein EM. Voluntary alteration of visual evoked potentials. Ann Neurol 1982; 12: 475-8.

3 Kramer KK, La Piana FG, Appleton B. Ocular malingering and hysteria. Surv Ophthalmol 1979; 24: 89-96.

4 Miller BW. A review of practical tests for ocular malingering and hysteria. Surv Ophthalmol 1973; 17: 241-6.

5 Quam K. Ocular malingering. Am Orthopt J 1974; 24: 73-6.

6 Thompson HS. Functional visual loss. Am J Ophthalmol 1985; 100: 209-13.

7 Teller DY, Morse R, Borton R, Regal D. Visual acuity for vertical and diagonal gratings in human infants. Vision Res 1974; 14: 1433-9.

8 McDonald MA, Dobson V, Sebris SL, Baitch L, Varner D, Teller DY. The acuity card procedure: a rapid test of infant acuity. Invest Ophthalmol Vis Sci 1985; 26: 1158-62.

9 Mohn G, van Hof-van Duin J, Fetter WPF, de Groot L, Hage M. Acuity assessment in non-verbal infants and children: clinical experience with the acuity card procedure. Dev Med Child Neurol 1988; 30: 232-44.

Accepted for publication 1 December 1988. 